\title{
Wideband and Ultrawideband Channel Models in Working Machine Environment
}

\author{
Attaphongse Taparugssanagorn, Matti Hämäläinen, and Jari Iinatti \\ Centre for Wireless Communications, University of Oulu, 90570 Oulu, Finland \\ Correspondence should be addressed to Attaphongse Taparugssanagorn, attaphongset@gmail.com
}

Received 23 April 2012; Revised 17 July 2012; Accepted 30 July 2012

Academic Editor: Matthias Pätzold

Copyright (C 2012 Attaphongse Taparugssanagorn et al. This is an open access article distributed under the Creative Commons Attribution License, which permits unrestricted use, distribution, and reproduction in any medium, provided the original work is properly cited.

\begin{abstract}
We present statistical models for wideband and ultrawideband (UWB) radio channels in a working machine cabin environment. Based on a set of measurements, it was found that such a small and confined space causes mostly diffuse multipath scattering rather than specular paths. The amplitude of the channel impulse responses in the wideband case is mostly Rayleigh distributed small-scale fading signal, with only a few paths exhibiting Ricean distributions, whereas the ones in the UWB case tend to be log-normally distributed. For the path amplitude, we suggest an exponential decay profile, which has a constant slope in $\mathrm{dB}$ scale, with the corresponding parameters for the UWB case. For the wideband case, a twofold exponential decay profile provides excellent fits to the measured data. It was also noted that the root-mean-square (RMS) delay spread is independent of the line-ofsight/obstructed line-of-sight situations of the channel. The multipath components contributing significant energy play a major role in such a small environment if compared to the direct path. In addition, the radio channel gains are attenuated with the presence of a driver inside the cabin.
\end{abstract}

\section{Introduction}

A wireless sensor network (WSN), which is a wireless network consisting of spatially distributed autonomous devices using sensors to cooperatively monitor physical or environmental conditions [1], today covers very widely in many industrial and civilian application areas, including industrial process monitoring and control, health monitoring, environment and habitat monitoring, home automation, and traffic control. Wireless intra-vehicle communications system has been proposed as a new type of communications system that can provide onboard passengers with bidirectional highspeed data exchange services for both communications and entertainment $[2,3]$. For the purpose of safety, comfort and convenience, WSNs are being deployed in the new models of automotives to collect such information as temperature, speed, pressure, and so on. This idea of exploiting WSNs is not limited to applications in a working machine only. The information and communication system used in a working machine makes it easier for driver to control the operation, for example, in a wood processing guiding the trunk cutting according to orders input from the system. This can guarantee quick processing during logging, and high productivity. This information system is expected to be wirelessly controlled by the driver. Using wireless communication links instead of wired link can save a big amount of installing costs. In addition, measuring vibrations acting on the human body, so-called "human vibration," is the prevention of health risks and the evaluation of comfort, for example, in vehicles [4]. Wireless vibration sensing application embedded to wireless body area networks (WBANs) can also be included in the future system.

The design of information and communication systems requires a good understanding of the corresponding radio wave propagation channel. The characteristics of an indoor radio propagation is found to be its site-specific behavior. Many existing radio channel measurement campaigns and the corresponding channel models have been restricted to typical residential and office environments, which are highly limited by the attenuation of walls $[5,6]$. Only few radio channel measurements were done inside a vehicle $[7,8]$. To our knowledge, there is no work done in a working machine environment, which can have the different radio channel characteristics due to its small and confined space. 
This paper discusses on experimental radio channel measurements that are carried out in a working machine environment within two different frequency bands, that is, the industrial, scientific, and medical (ISM) radio frequency band $(2.4-2.4835 \mathrm{GHz})$ and the ultrawideband (UWB) frequency band $(3.1$ and $10 \mathrm{GHz}$ ). The statistical radio channel models based on the measurement results are then developed. The novelty of this paper comes from the new environment whose radio channel characteristics are modeled.

Section 2 of the paper is devoted to the description of the measurement setup including the scenarios. Section 3 addresses the characterization of the measured radio channels. The average channel impulse responses for each scenario are analyzed and discussed. The parameters of the statistical channel model are also presented in this section. Section 4 deals with the implementation of the channel model and its evaluation. The conclusions of this work are given in Section 5.

\section{Measurement Campaigns}

2.1. Measurement Setup. The measurements were carried out in a frequency domain using Agilent 8720ES S-parameter vector network analyzer (VNA) [9]. The network analyzer is operated in a transfer function measurement mode, where port 1 and port 2 are the transmitting and the receiving ports, respectively. Thus, the measurements result as a frequency response of the channel.

The measurements were carried out in the frequency band between $2.4-2.4835 \mathrm{GHz}$, which is in the ISM band, and is used, that is, by the standards IEEE 802.11 and 802.15.4 dedicated for wireless local area network (WLAN) communication. The measurements in an UWB frequency band between 3.1-10 GHz were also conducted. Therefore, the measured bandwidths $B$ were $83.5 \mathrm{MHz}$ and $6.9 \mathrm{GHz}$, respectively. Consequently, the corresponding delay resolutions are $11.97 \mathrm{~ns}$ and $0.14 \mathrm{~ns}$. The maximum number of frequency points per sweep $M$ is 1601, which can then be used to calculate the maximum detectable delay $\tau_{\max }$ of the channel as

$$
\tau_{\max }=\frac{(M-1)}{B} .
$$

Using (1), the maximum detectable delay, $\tau_{\max }$ of the channels for two different bandwidths are $19.16 \mu$ s and $231.88 \mathrm{~ns}$, which correspond to $5.75 \mathrm{~km}$ and $69.56 \mathrm{~m}$, respectively, in a free-space distance. Maximum detectable delay is just a measurement system-based parameter. It is introduced to show that the measurement system is able to detect all propagation paths having significant contribution to the received power. However, the usable signal energy is in the shorter delays than the maximum detectable delay as can be seen later on.

The antennas for the measurements in WLAN band 2.4$2.4835 \mathrm{GHz}$ were HGA7S $7 \mathrm{dBi}$ high gain antennas [10]. The SkyCross SMT-3TO10 M-A antennas [11] were used for the measurements in UWB band. Both types of the antennas are linearly polarized. The SkyCross SMT-3TO10 M-A antennas
TABLE 1: Measurement system parameters.

\begin{tabular}{lc}
\hline Parameters & Value \\
\hline Frequency band & ISM $(2.4-2.4835 \mathrm{GHz})$, \\
& UWB $(3.1-10 \mathrm{GHz})$ \\
Bandwidth & $83.5 \mathrm{MHz}$, \\
& $6.9 \mathrm{GHz}$ \\
Number of sample points within the & 1601 \\
band & $19.16 \mu \mathrm{s}(5.75 \mathrm{~km})$, \\
Maximum detectable delay & $231.88 \mathrm{~ns}(69.56 \mathrm{~m})$ \\
Sweep time & $800 \mathrm{~ms}$ \\
Average noise floor & $-108 \mathrm{dBm}$ \\
Transmit power & $5 \mathrm{dBm}$ \\
Tx and Rx cables loss & $7.96 \mathrm{~dB}$ \\
\hline
\end{tabular}

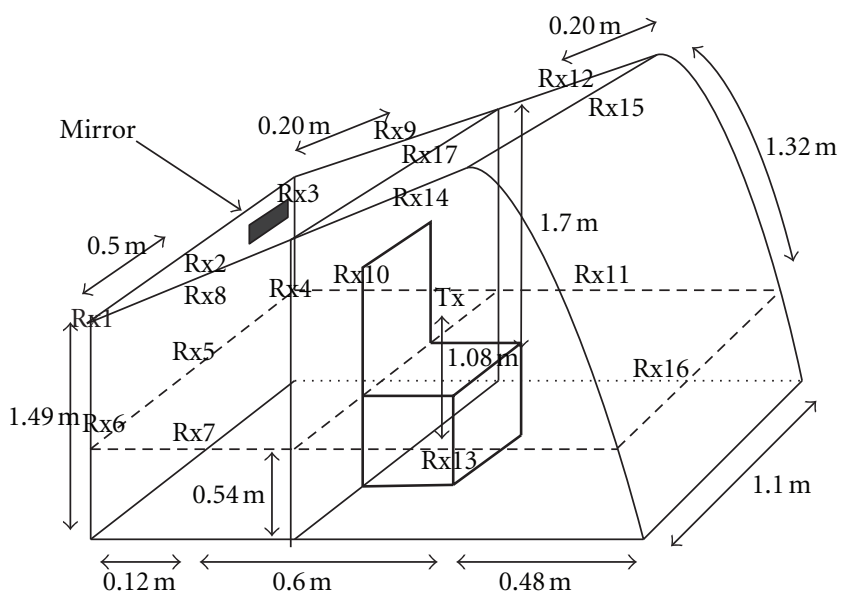

Figure 1: Arrangement of the antennas' positions for measurements inside a working machine cabin.

are azimuthally omnidirectional, whereas the HGA7S $7 \mathrm{dBi}$ high gain antennas are designed for 360 degrees of coverage [10]. The sweep time of the VNA depends on the frequency points within the sweep band, being automatically adjusted by the network analyzer, that is, radio channels were measured in a frequency domain over the frequency band of interest. This corresponds to a conventional $S_{21}$ parameter $\left(S_{21}\right.$ parameter refers to the signal exiting at Port 2 for the signal incident at Port 1 of VNA) measurement setup, where the radio channel is the device under test (DUT). The transmit power used in this study was $5 \mathrm{dBm}$ (equivalent to $3.16 \mathrm{~mW}$ ) as the VNA output port. Table 1 summarizes the measurement system parameters.

2.2. Channel Measurement Description. The measurements were conducted inside a cabin of a working machine with the dimensions as illustrated in Figure 1. The transmit (Tx) antenna and the receive $(\mathrm{Rx})$ antenna are, in general, set to face each other, and the line-of-sight (LOS) path always exists except when the links are blocked by the seat. The arrangement of the antennas' positions is shown in Figure 1. The position of the Tx antenna is fixed at the 
seat on $1.08 \mathrm{~m}$ above the cabin floor. The positions of the $\mathrm{Rx}$ antenna were moved to 17 different spots, namely, from the position Rx1 till the position Rx17. At each position, 100 consecutive measurements of the channel were taken to improve statistical reliability. This number of typically used in static cases. In order to study radio channels inside a cabin in a working machine for real situations, the following three scenarios are investigated:

(i) scenario 1: without a driver, when the engine is off.

(ii) scenario 2: with a driver sitting, when the engine is off.

(iii) scenario 3: with a driver sitting, when the engine is on.

\section{Channel Measurement Results and Data Analysis}

Measured $S_{21}$-parameters, that is, channel transfer functions, are converted to the time domain, that is, to channel impulse responses $h(\tau)$, using an inverse fast Fourier transform (IFFT). A Hamming window is used to reduce sidelobes. The channel impulse response is generally given by a tapped delay line model [12] as

$$
h(\tau)=\sum_{l=0}^{L-1} a_{l} \delta\left(\tau-\tau_{l}\right) \exp \left(j \phi_{l}\right),
$$

where $a_{l}$ is the amplitude of the $l$ th path, $\tau_{l}$ is the arrival time of the $l$ th path, $\phi_{l}$ is the phase for the $l$ th path, and $L$ is the number of paths. $\delta(t)$ stands for the Dirac function, which is the impulse symbol and is sometimes called as "Dirac's delta function." The phase $\phi_{l}$ is modeled by a uniform distribution $U\{0,2 \pi\}$. The statistical parameters defining the channel model are presented in the following paragraphs.

3.1. Average Channel Impulse Response. One hundred individual realizations of the channel impulse response were averaged for each position. The reason for using one hundred realizations is since this is typically used to guarantee statistical reliability. This number of sweeps is recorded for all the individual links measured, so the total number of sweeps carried out during the measurement campaign is much higher. When using frequency domain measurement technique, the environment needs to be static, which means that one hundred consecutive measurements per link is enough.

To proceed further analysis, all measured channel impulse responses are first truncated above the noise threshold, set to the noise level adding $10 \mathrm{~dB}$ (about $-98 \mathrm{dBm}$ ). The only paths above the noise threshold are taken into account. Some examples are presented here in order to explain the analysis. The large-scale features (i.e., path loss) and also small-scale features (due to the change in pulse shape) of the channels are taken into account. The large-scale models are necessary for network planning and link budget design while small-scale models are necessary for efficient receiver design and performance analysis. This kind of models is not

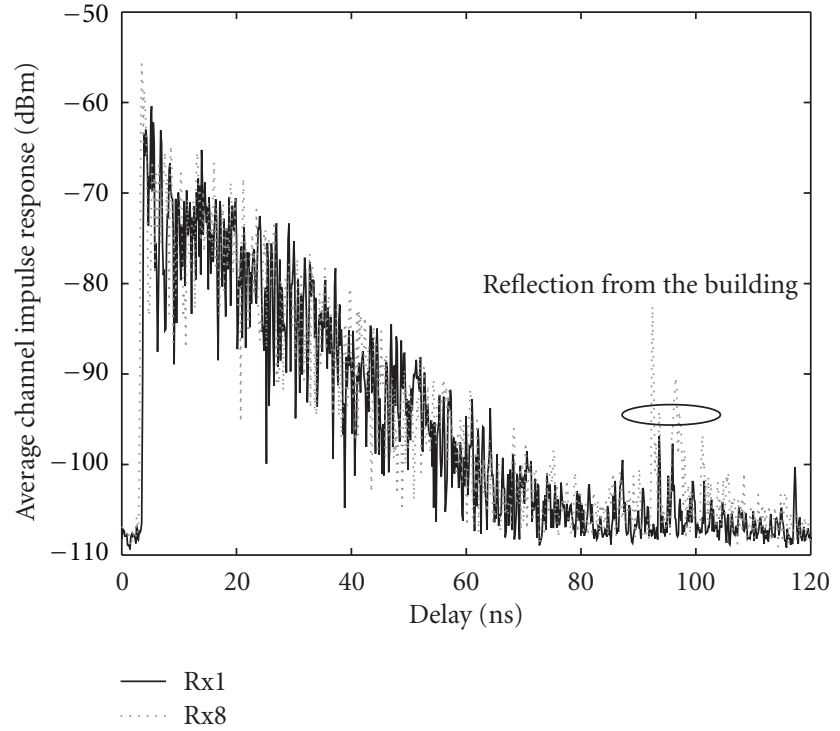

Figure 2: Average channel impulse responses for the UWB band in scenario 1: without a driver, when the engine is off. The positions of $\mathrm{Rx}$ antenna are $\mathrm{Rx} 1$ and $\mathrm{Rx} 8$.

publicly available at open literature for working machine's cabin environment so far.

As preliminarily shown in [13], the measured channel impulse responses for the ISM band have two constant slopes in $\mathrm{dB}$ scale, which correspond to a typical exponential decay profile in a linear scale. This shows that there are many reflections rather than a significant specular path. This is excellently suited to characterize diffuse scattering [14].

The average channel impulse responses for the UWB band (6.9 GHz bandwidth) in scenario 1 , without a driver, when the engine is off shown in Figure 2 were also represented in [13]. The UWB measurements allow us to check the presence of many paths arriving at the receiver within the delay resolution and the maximum delay. Firstly, the channel impulse responses have a constant slope in $\mathrm{dB}$, which corresponds to a typical exponential decay profile in a linear scale. The other advantage of the UWB measurements is the high temporal resolution, which makes it possible to see the first path more precisely. The first path arrival time can approximately translate into the distance between $\mathrm{Tx}$ and $\mathrm{Rx}$ antennas. Therefore, the distances between $\mathrm{Tx}$ and $\mathrm{Rx} 1, \mathrm{Rx} 3, \mathrm{Rx} 8$ as well as $\mathrm{Rx} 17$ are $1.17 \mathrm{~m}, 1.35 \mathrm{~m}, 1.05 \mathrm{~m}$, and $0.81 \mathrm{~m}$, respectively $(3.9 \mathrm{~ns}, 4.5 \mathrm{~ns}, 3.5 \mathrm{~ns}$, and $2.7 \mathrm{~ns}$, resp.). The Rx1 and $\mathrm{Rx} 3$ are in the obstructed line-of-sight (OLOS) case; thus the first path is not the strongest path. Depending on the position, the constructive multipaths can create a stronger path to the receiver. The $\mathrm{Rx} 8$ and $\mathrm{Rx} 17$ are LOS cases; therefore, the first path is also the strongest path. Figure 3 illustrates the average measured channel impulse responses for the ISM band, Rx5 (OLOS), in three different scenarios. When the driver is inside the cabin, the radio waves in all multipaths from the Tx close to the body of the driver $(\approx 3 \mathrm{~cm})$ are attenuated. In addition, the first path arrival time is longer than the one in the case of no driver inside the cabin. 


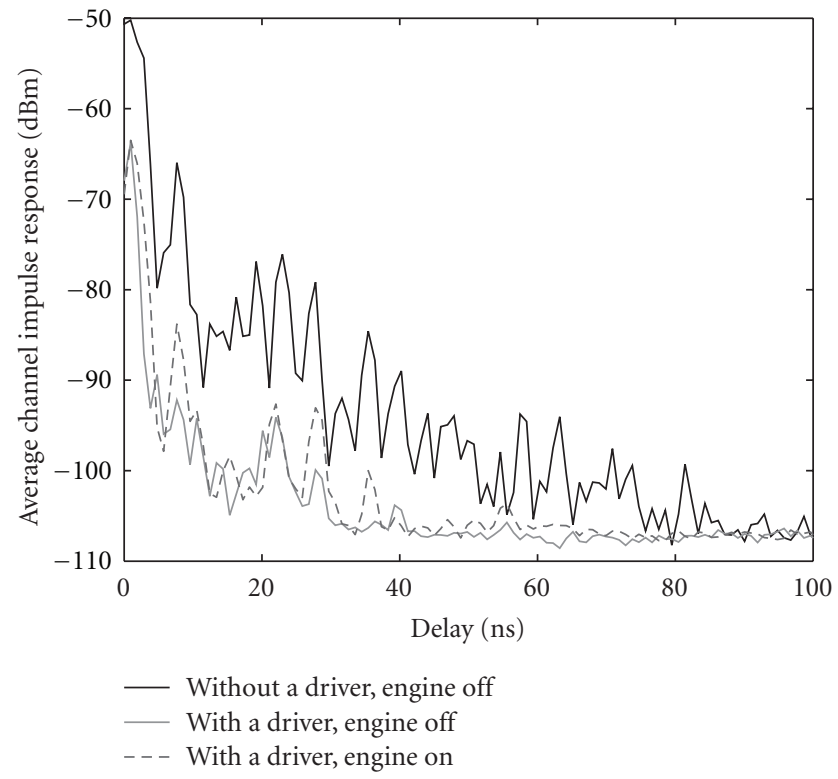

FIGURE 3: Average measured channel impulse responses for the ISM band, Rx5 (OLOS), in three different scenarios.

Note that in the following, the analysis has been carried out against several distributions, and the best fits have been selected. Moreover, all the parameters used in the models were extracted from the measured data during the postprocessing. From the extracted parameters, we develop measurements-based deterministic channel models for both ISM band and UWB band cases.

3.1.1. Exponential Decaying Factor $\left(a_{l}\right)$. As explained above, the path amplitude $a_{l}$ in (2) is approximately modeled by an exponential decay profile with a Ricean factor $\gamma_{0}$ and an exponential decaying factor $\Gamma$ for UWB case as

$$
10 \log _{10}\left|a_{l}\right|= \begin{cases}0, & l=0 \\ \gamma_{0}+10 \log _{10}\left(\exp \left(\frac{-t_{l}}{\Gamma}\right)\right), & 1 \leq l \leq L-1 .\end{cases}
$$

For ISM case, the channel impulse responses have two constant slopes in $\mathrm{dB}$, and thus the path amplitude $a_{l}$ in (2) is approximately modeled by twofold exponential decay profile with two Ricean factors $\gamma_{01}$ and $\gamma_{02}$ as well as two exponential decaying factors $\Gamma_{1}$ and $\Gamma_{2}$ as

$$
10 \log _{10}\left|a_{l}\right|= \begin{cases}0, & l=0 \\ \gamma_{01}+10 \log _{10}\left(\exp \left(\frac{-t_{l}}{\Gamma_{1}}\right)\right), & 1 \leq l \leq l_{1} \\ \gamma_{02}+10 \log _{10}\left(\exp \left(\frac{-t_{l}}{\Gamma_{2}}\right)\right), & l_{1}+1 \leq l \leq L-1,\end{cases}
$$

where $l_{1}$ is the last path of the first part.

An example from each frequency band is presented in Figure 4 showing the average channel impulse response for the ISM band and for Rx17 (LOS) in the first scenario

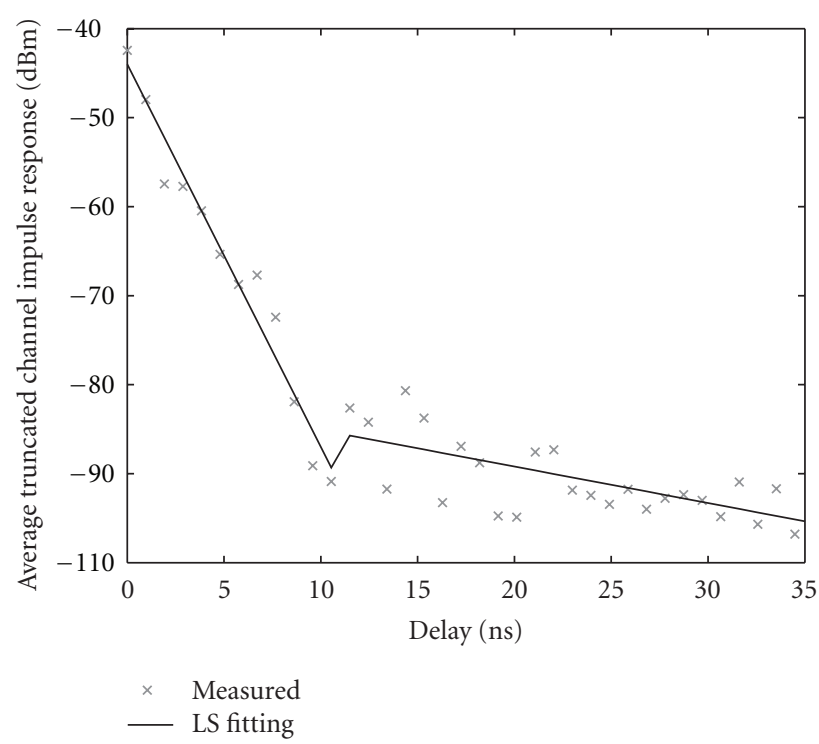

FIGURE 4: Average channel impulse responses for the ISM band for Rx17 (LOS) and the least square linear fitting curve in dB scale.

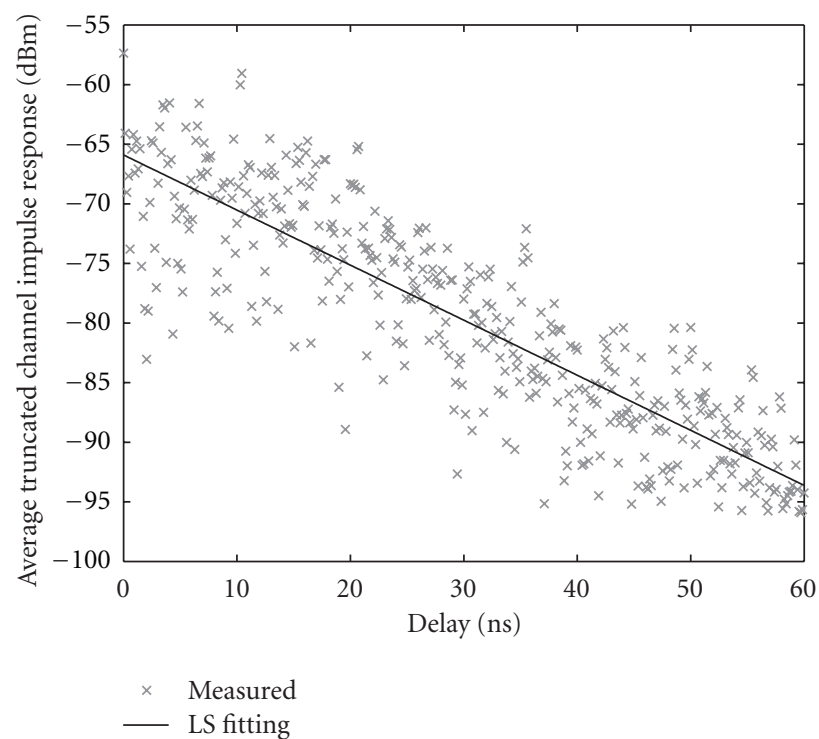

FIGURE 5: Average channel impulse responses for the UWB band for $\mathrm{Rx} 17$ (LOS) and the least square linear fitting curve in $\mathrm{dB}$ scale.

(without a driver, the engine is off) and the twofold least square (LS) linear fitting curve in $\mathrm{dB}$ scale. The corresponding factors $\gamma_{01}$ and $\gamma_{02}$ are $-44 \mathrm{~dB}$ and $-81 \mathrm{~dB}$, respectively, as well as the exponential decaying factor $\Gamma_{1}$ and $\Gamma_{2}$ are 2.33 and 24.39 , respectively. Figure 5 shows the average channel impulse response for the UWB band and for Rx17 (LOS) and the LS linear fitting in $\mathrm{dB}$. The corresponding factor $\gamma_{0}$ and the exponential decaying factor $\Gamma$ are $-66 \mathrm{~dB}$ and 21.74 .

3.1.2. Amplitude Variation ( $\chi$ ). This part represents the amplitude variation $\chi$ over the mean value of the path amplitude. The cumulative distribution functions (CDFs) in Figures 6 and 7 depict that the amplitude variations of the 

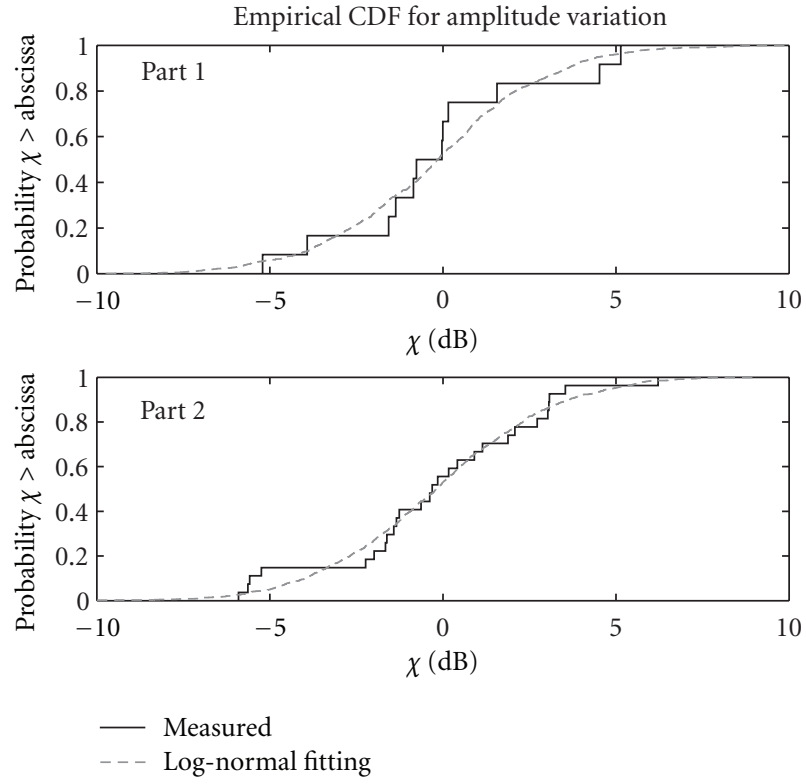

FIGURE 6: CDFs of the amplitude variation over the mean value of the path amplitude and the log-normal fitting curve for the ISM band and for Rx17 (LOS).

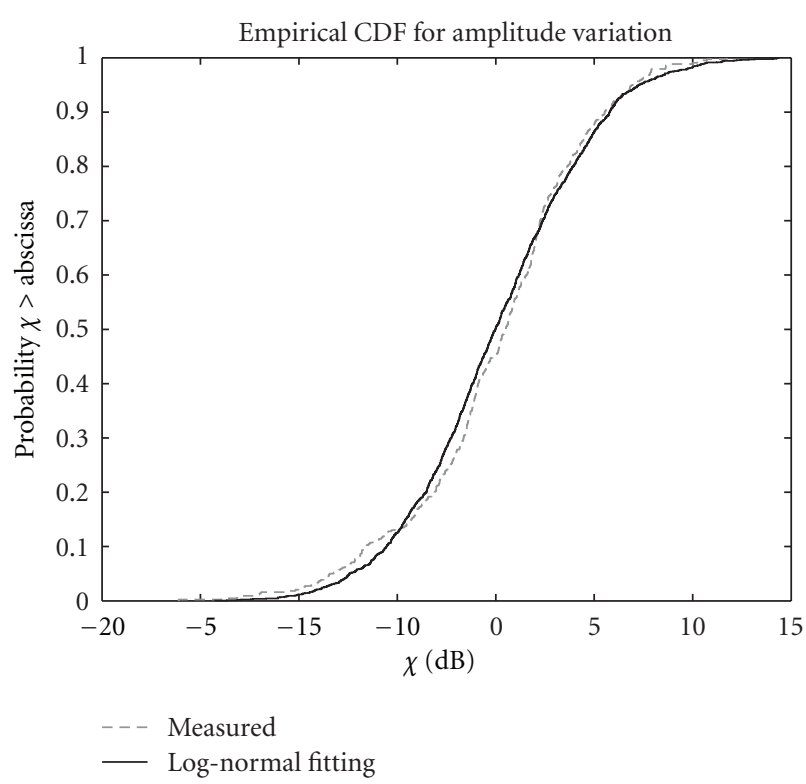

FIGURE 7: CDFs of the amplitude variation over the mean value of the path amplitude and the log-normal fitting curve for the UWB band and for Rx17 (LOS).

average channel impulse responses are well fitted by the lognormal distribution with zero mean and standard deviation $\sigma_{\chi}$ of $6.69 \mathrm{~dB}$ and $4.50 \mathrm{~dB}$, respectively.

3.1.3. Number of Paths $(L)$. The CDFs of the number of paths $L$ (above the noise threshold) are excellently fitted by Poisson distribution with a certain mean $\mu_{L}$. An example for Rx14 (LOS) in the UWB band shown in Figure 8, respectively. The number of paths decreases with the presence of the driver. In the case of the ISM band, the CDFs of the number of

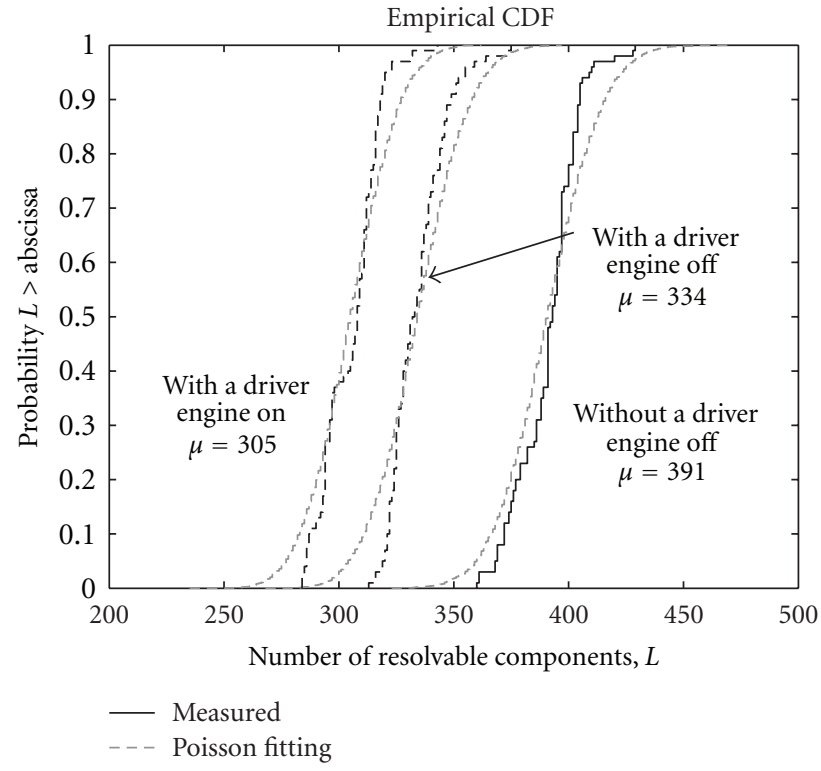

FIgURE 8: CDFs of the number of paths $L$ for Rx14 (LOS) in the UWB band measurements.

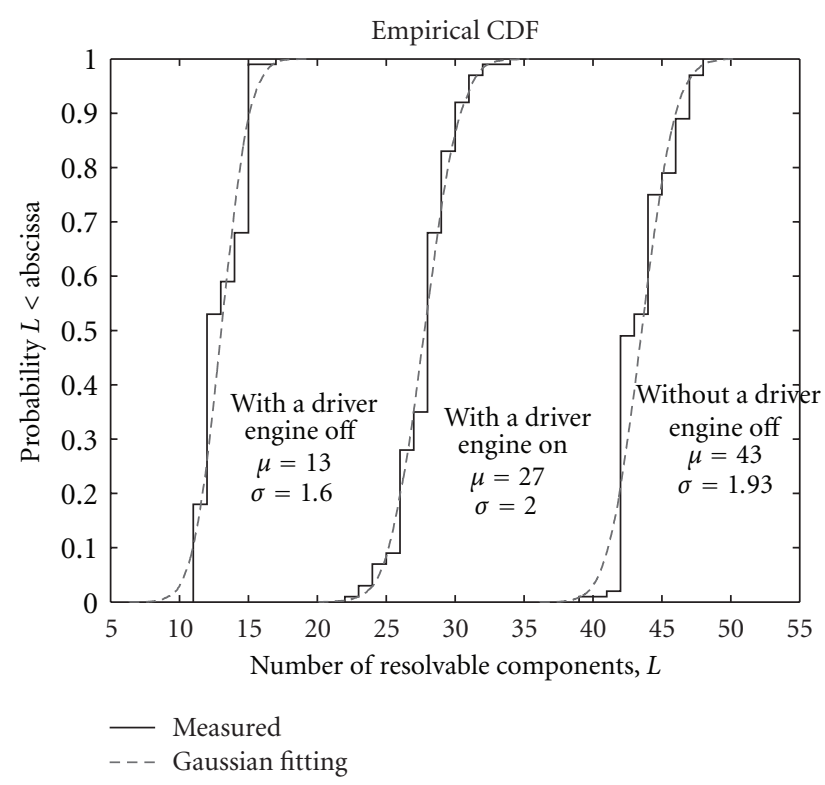

FIGURE 9: CDFs of the number of paths $L$ for Rx14 (LOS) in the ISM band measurements.

paths $L$ are fitted by Gaussian distribution with certain mean $\mu_{L}$ and standard deviation $\sigma_{L}$ as an example in Figure 9 for Rx14 (LOS). The number of paths presented in the paper is for channel modeling purpose. Nonetheless, they are much more than the typical number of paths in system design, for example, the number of paths within $10 \mathrm{~dB}$ of the strongest path and the number of paths containing 85 percent of the energy, which give approximately $35-50$ paths in the UWB band case. As can be seen, the impact of the vibration (of the engine) on the number of the paths is clear, especially, in the case of OLOS unlike the other channel characteristics. 


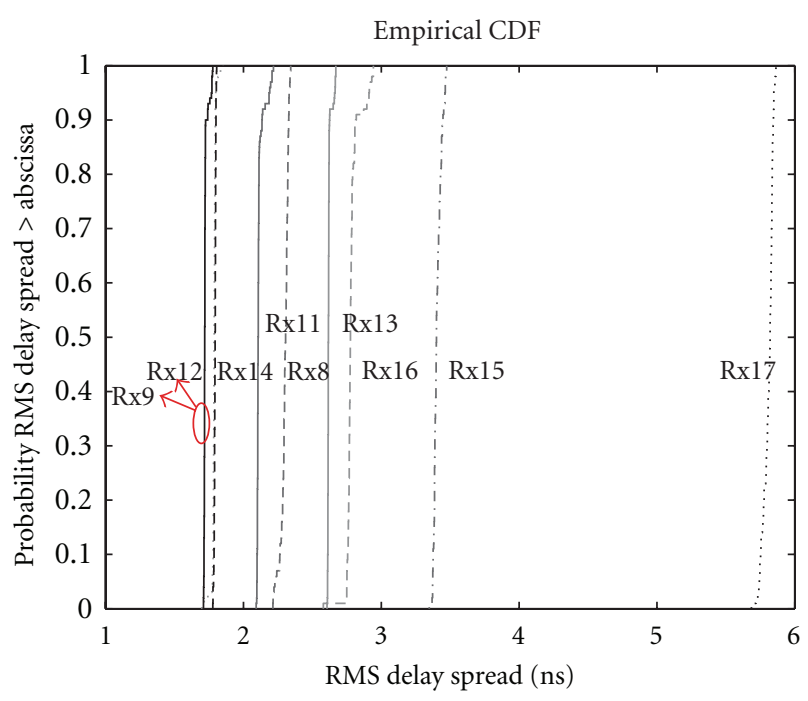

FIGURE 10: CDFs of the measured RMS delay spreads for LOS in the UWB band measurements.

\subsection{Small-Scale Channel Characterization}

3.2.1. Delay Dispersion. In order to compare different multipath channels and to develop general design guidelines, parameters which grossly quantify the multipath channel are used. The mean excess delay $\tau_{m}$ and root-mean-square (RMS) delay spread $\tau_{\text {RMS }}$ are commonly used to imply the time delay dispersive properties of a wideband multipath channel parameters that can be determined from a power delay profile. The received signal will suffer spreading in time if compared to the transmitted signal. This dispersion can also imply frequency selective fading and intersymbol interference (ISI). No serious ISI is likely to occur if the symbol duration is longer than, say, ten times the RMS delay spread [14]. The RMS delay spread is the standard deviation (or root-mean-square) value of the delay of reflections, weighted proportional to the energy in the reflected waves defined as [14]

$$
\tau_{\mathrm{RMS}}=\sqrt{\frac{\sum_{i=1}^{L}\left(\tau_{i}-\tau_{\mathrm{m}}\right)^{2} \cdot\left|h\left(\tau_{i}\right)\right|^{2}}{\sum_{i=1}^{L}\left|h\left(\tau_{i}\right)\right|^{2}}},
$$

where $\tau_{m}$ is expressed as

$$
\tau_{m}=\sqrt{\frac{\sum_{i=1}^{L} \tau_{i} \cdot\left|h\left(\tau_{i}\right)\right|^{2}}{\sum_{i=1}^{L}\left|h\left(\tau_{i}\right)\right|^{2}}} .
$$

Figures 10 and 11 depict the CDFs of the measured RMS delay spreads for LOS and OLOS situations, respectively, for the UWB band measurements. All values are in the range of $1.7-5.8 \mathrm{~ns}$, which are very small if compared to typical indoor RMS delay spread varying between 14 and $18 \mathrm{~ns}$ [14]. We can see that the RMS delay spread is independent of the LOS/OLOS situations of the channel. Neither of the distance, this is because the cabin space is very small. The multipath components contributing significant energy play a major role in such a small environment if compared to the direct path. This is very important on the selection of the antenna position and also to the receiver structure.

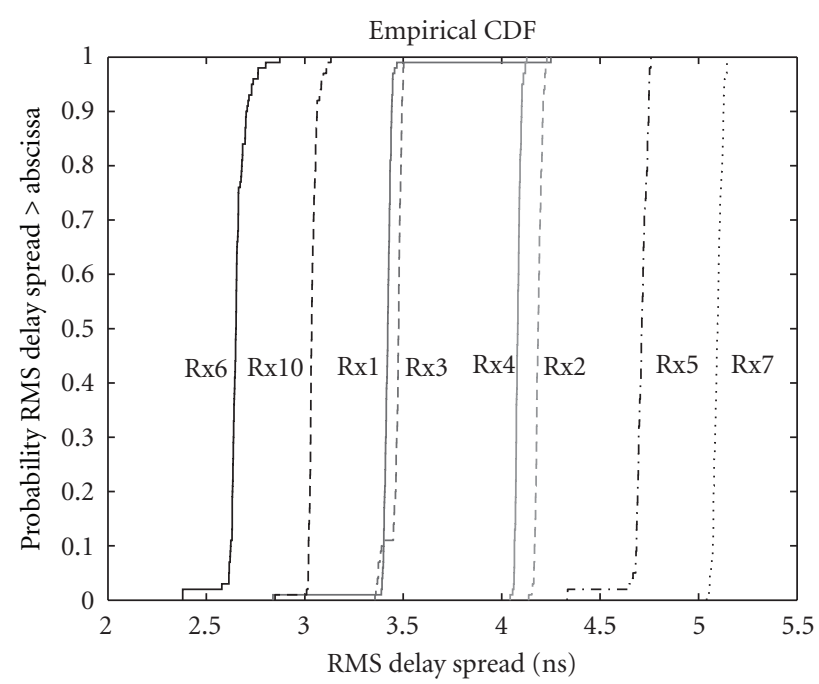

FIGURE 11: CDF of the measured RMS delay spreads for OLOS in the UWB band measurements.

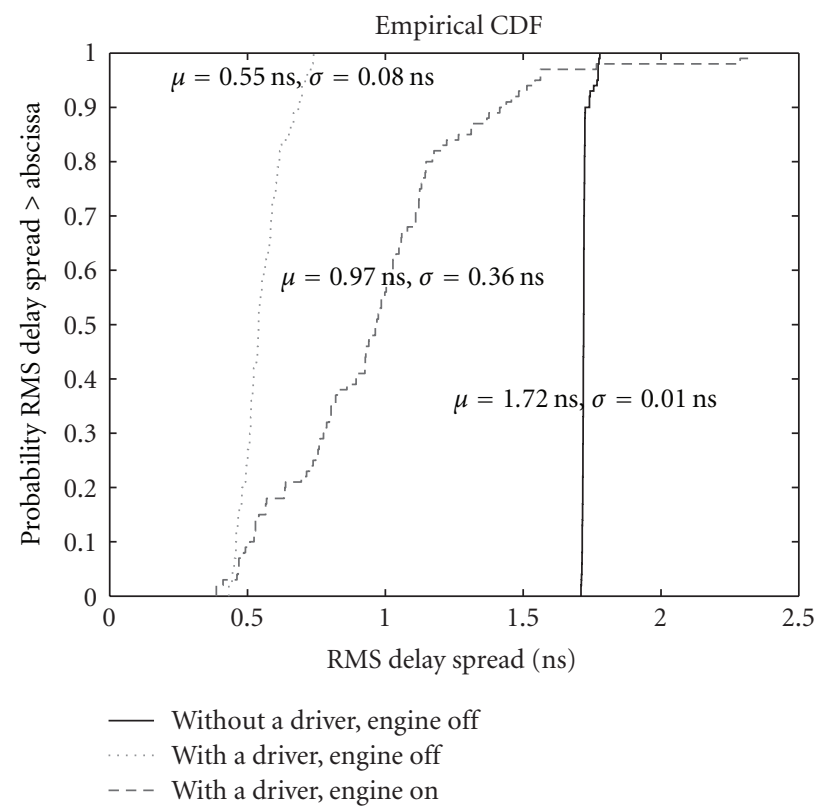

FIgURE 12: CDFs of the measured RMS delay spreads for Rx12 (LOS) in the UWB band measurements in all three scenarios.

An example for the case of the cabin with a driver, when the Rx antenna is at Rx12 (LOS), is shown in Figure 12. The RMS delay spread decreases by approximately $1.2 \mathrm{~ns}$, since the presence of people in the cabin attenuates the path gains. Corresponding CDFs for OLOS case $(\mathrm{Rx} 4)$ are shown in Figure 13. The standard deviations $\sigma$ of the RMS delay spreads, when the engine is on (cabin vibration), are larger than the ones, when the engine is off. This shows that the radio channels differ considerably for the case with and without driver.

3.2.2. Amplitude Distribution. In the ISM band, the power received for each delay tap is the vectorial sum of many multipath components arriving in the corresponding delay 


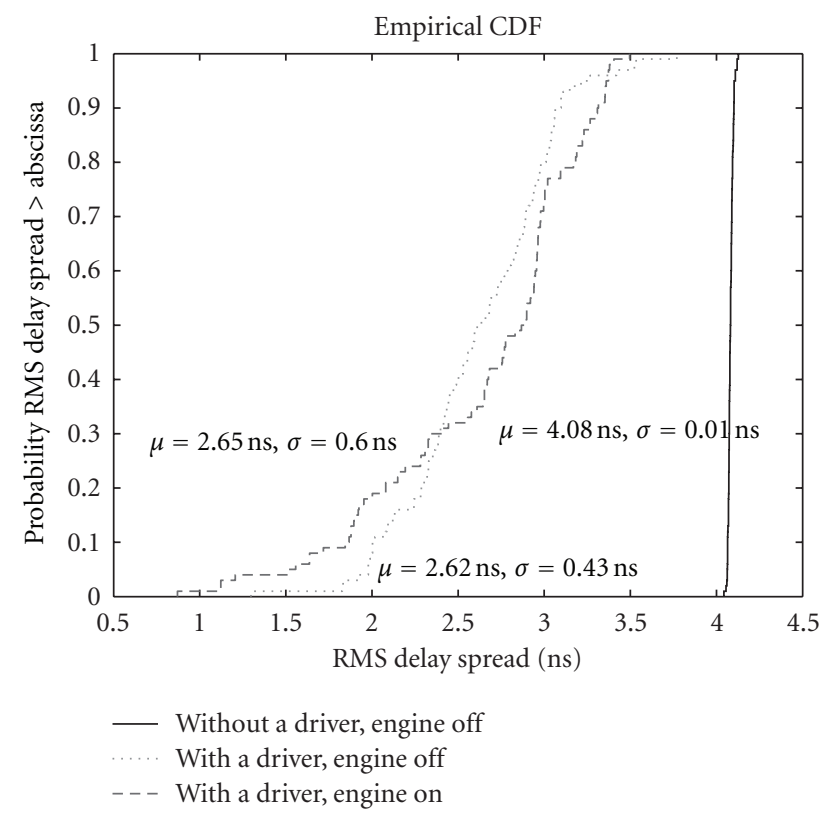

FIgURE 13: CDFs of the measured RMS delay spreads for $\mathrm{Rx} 4$ (OLOS) in the UWB band measurements in all three scenarios.

bin. The central limit theorem is therefore valid, and the amplitudes of the delay bins exhibit a Rayleigh (in nonLOS or OLOS case) or a Ricean (in LOS case) distribution [15]. On the contrary, only few multipath components (MPCs) fall into each resolvable delay in the UWB band. Therefore, the central limit theorem is not applicable and the amplitudes of the delay bins consequently do not exhibit a Rayleigh or Rice distributions.

In the ISM band case, in all scenarios, the first delay bins tend to be Ricean distribution due to their high $K$ factors [16], whereas the other delay bins can be assumed to be Rayleigh distributed due to their very small $K$ factors.

For LOS case in the UWB band, as explained above, only few MPCs fall into each resolvable delay and the central limit theorem is therefore not applicable. The measured amplitudes are rather log-normally distributed [17].

3.3. Large-Scale Channel Characterization. Path loss (PL) is an important large-scale characteristic for link budget calculation and system design. In the conventional narrowband channel, Friis transmission formula is used to model the received signal power, $P_{r}$ in free space given by [18]

$$
P_{r}(d)=\frac{P_{t} G_{t} G_{r} \lambda^{2}}{(4 \pi d)^{2}},
$$

where $P_{t}$ is the transmitted power, and $G_{t}$ and $G_{r}$ are the $\mathrm{Tx}$ and the $\mathrm{Rx}$ antenna gains, respectively. $\lambda$ is the wavelength, and $d$ is the Tx-Rx separation distance. The Friis transmission formula shows that the received signal power falls off as the square of the $\mathrm{Tx}-\mathrm{Rx}$ separation distance. From (7), the term PL is given by $(\lambda / 4 \pi d)^{2}$ which predicts that signal power will decrease with the square of increasing frequency and distance. These show the presence of both distance and frequency dependency in path loss.
TABLe 2: Average path loss (PL) within the measured band.

\begin{tabular}{lc}
\hline Measurement scenarios and cases & Mean path loss $(\mathrm{dB})$ \\
\hline UWB band & $73.31 \mathrm{~dB}$ \\
Scenario 1, LOS & $77.32 \mathrm{~dB}$ \\
Scenario 1, OLOS & $75.86 \mathrm{~dB}$ \\
Scenario 2, LOS & $79.90 \mathrm{~dB}$ \\
Scenario 2, OLOS & $76.20 \mathrm{~dB}$ \\
Scenario 3, LOS & $80.16 \mathrm{~dB}$ \\
Scenario 3, OLOS & \\
ISM band & $66.74 \mathrm{~dB}$ \\
Scenario 1, LOS & $71.85 \mathrm{~dB}$ \\
Scenario 1, OLOS & $80.20 \mathrm{~dB}$ \\
Scenario 2, LOS & $90.24 \mathrm{~dB}$ \\
Scenario 2, OLOS & $80.82 \mathrm{~dB}$ \\
Scenario 3, LOS & $90.92 \mathrm{~dB}$ \\
Scenario 3, OLOS & \\
\hline
\end{tabular}

Nevertheless, the frequency is constant within a bandwidth of interest and is thus negligible. However, for UWB systems, the frequency dependency in PL can be significant due to the large bandwidth ( $>500 \mathrm{MHz}$ ). The channel would distort the signal spectrum of the signal which is directly related to the signal distortions, that is, pulse shape distortion. PL can be obtained directly from the measured channel transfer function $H(f)$. The distance dependency of the path loss is calculated by time snapshot and frequency averaging over the total bandwidth at each $\mathrm{Rx}$ position as

$$
\operatorname{PL}(d)=\frac{1}{M N} \sum_{i=1}^{N} \sum_{j=1}^{M}\left|H\left(f_{i}, t_{j} ; d\right)\right|^{2},
$$

where $H\left(f_{i}, t_{j} ; d\right)$ denotes the $j$ th channel transfer function time snapshot at frequency $f_{i}$ in a distance $d$. $N$ and $M$ denote the number of sample points within the band and the number of snapshots, respectively.

The mean PLs for both LOS and OLOS cases are calculated from (8) and averaged over the results from all $\mathrm{Rx}$ positions in each case. The results for each case and each scenario are summarized in Table 2. As expected, the PL increases in the OLOS case and with the presence of a driver. Moreover, it is noted that the PL in the case when the engine is on is not very different than in the case when the engine is off. This follows the initial assumption.

\section{Channel Modeling and Evaluation}

In this section, we describe an experimental based deterministic channel model which are developed for both ISM band and UWB band cases. As shown in Figures 14 and 15 in Section 3.2.2, the amplitude of the first five delay bin in the ISM band case for the LOS case tends to be Ricean distributed. The remaining delay bins can be assumed to be Rayleigh distributed due to their small $K$-factors. For the OLOS case, only the first delay bin tends to be Ricean distributed, whereas the rest can be assumed to be Rayleigh distributed. In the UWB case, the amplitudes of all delay 


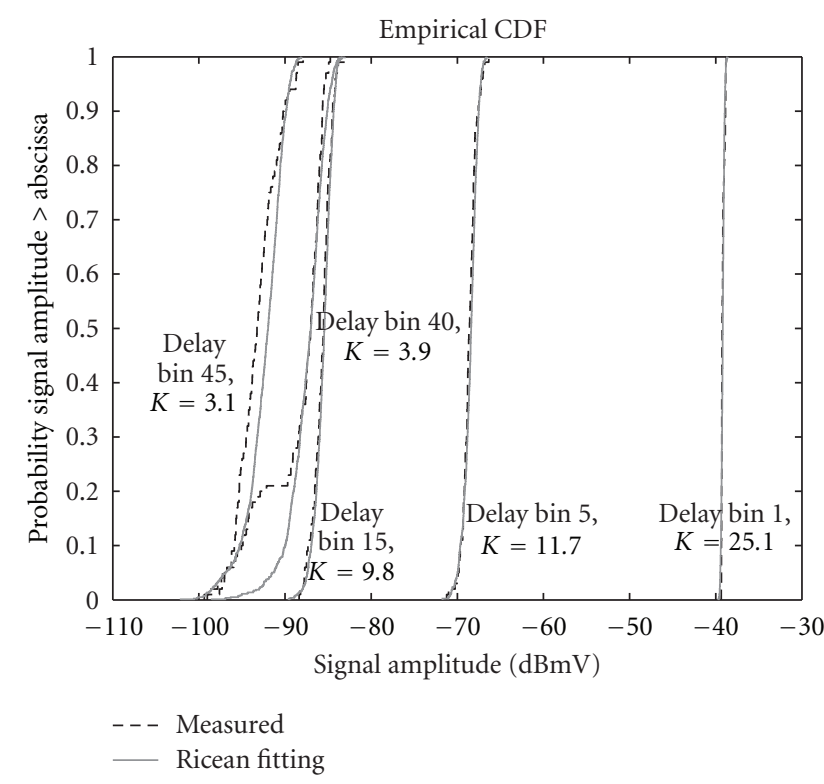

Figure 14: CDFs of the amplitudes of five delay bins, 1, 5, 15, 40 and 45 with corresponding $K$ factors for LOS case at Rx12.

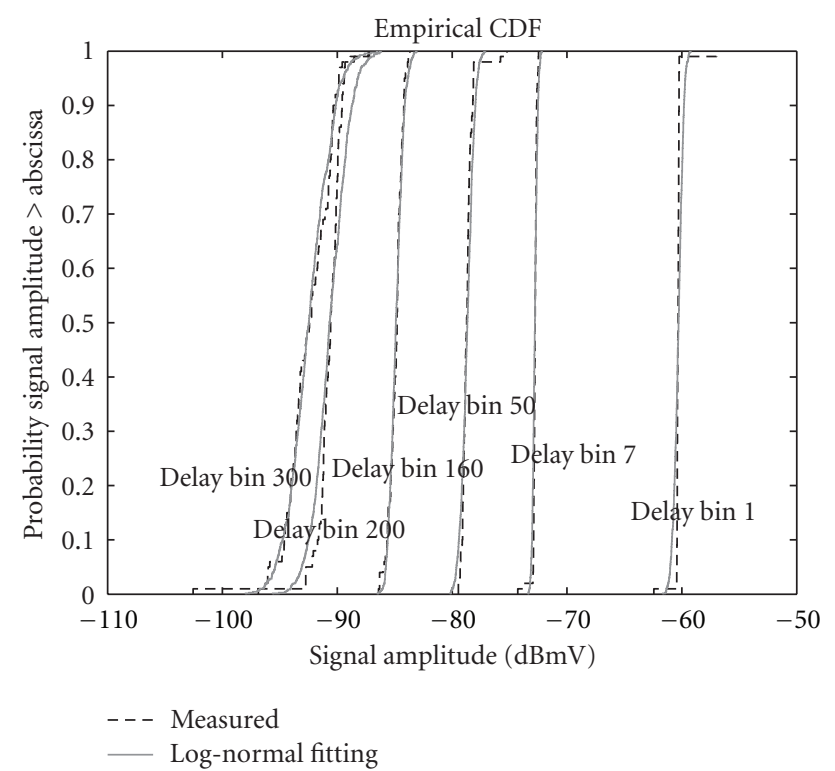

FIGURE 15: CDFs of the amplitudes of four delay bins, 1, 5, 15, and 40 with log-normal fitting for OLOS case at Rx1, without a driver and the engine off.

bins tend to be log normally distributed. To achieve that a random vector $\mathbf{x}$, where each component is the value of each delay bin with the corresponding distribution, either Ricean or Rayleigh or log normal up to the cases, with zero $(\mathrm{dB})$ mean is generated. Subsequently, the amplitude vector of the channel $|\mathbf{h}|$ is obtained by scaling the random vector with an exponential decay profile with the corresponding Ricean factor $\gamma_{0}$ and exponential decaying factor $\Gamma$ described in Section 3.1.1. Finally, the phase vector of the channel $\Phi$ is assumed to be uniformly distributed $U\{0,2 \pi\}$.

As an example, the channels for the case of Rx17 (LOS) in the ISM band and the case of Rx17 (LOS) in the UWB
TABLE 3: Channel parameters for simulation for the case of $\mathrm{Rx} 17$ (LOS).

\begin{tabular}{lcc}
\hline Parameters & ISM band & UWB band \\
\hline$\mu_{L}$ (paths) & 53 & 441 \\
$\gamma_{01}(\mathrm{~dB})$ & -44 & - \\
$\gamma_{02}(\mathrm{~dB})$ & -81 & - \\
$\gamma_{0}(\mathrm{~dB})$ & - & -66 \\
$\Gamma_{1}$ & 2.33 & - \\
$\Gamma_{2}$ & 24.39 & - \\
$\Gamma$ & - & 21.74 \\
& Ricean with $K$-factor & log normal \\
Amplitude & 21.35, 16.70, 16.28, 14.40, & for all delay bins \\
distribution & and 13.89 dB for the delay & \\
& bin 1-5, Rayleigh for the & \\
$\sigma_{\chi}(\mathrm{dB})$ & remaining delay bins & \\
& 2.97 and 3.04 & 4.50 \\
\hline
\end{tabular}

band (Figures 4 and 5) are simulated. For the former case, it is done by using (3) and the simulation parameters presented in Table 3. For the latter case, (4) is used with the simulation parameters in Table 3.

The average channel impulse response and the RMS delay spread $\tau_{\text {RMS }}$ are used for validating the simulated model as depicted in Figures 16 and 17. As we can see, the simulated channels have a good agreement with the measured channels.

\section{Conclusions}

In order to deploy wireless information and communication systems in such a confined environment like in a cabin of a working machine, a good understanding of the radio propagation channels, and a simple corresponding channel model are required. To our knowledge, there is no work done in a working machine environment, which have different radio channel characteristics due to its small and confined space. The measurement campaigns inside the working machine cabin were taken for two different frequency ranges, that is, in $2.4-2.4835 \mathrm{GHz}$ ISM band used, for example, by the standards IEEE 802.11 and 802.15.4 dedicated for wireless local area network communication, as well as, in an ultra wideband frequency band 3.1-10 GHz. Three different scenarios for each frequency band, that is, (1) without a driver, the engine is off, (2) with a driver, the engine is off, and (3) with a driver, the engine is on, were carried out.

The characteristics of the propagation channels were quite different from a traditional indoor environment; namely, there are many reflections rather than a significant specular path. This is excellently suited to characterize diffuse scattering. The UWB measurements have the high temporal resolution advantage, which consequently makes us able to see the first path accurately. This is translated into the distance between $\mathrm{Tx}$ and $\mathrm{Rx}$ antennas. With the presence of the driver inside the cabin, the radio channel gains are attenuated. In addition, the first path arrival time comes later than the one in the case of no driver inside the cabin. There was no significant difference found between the cases when 


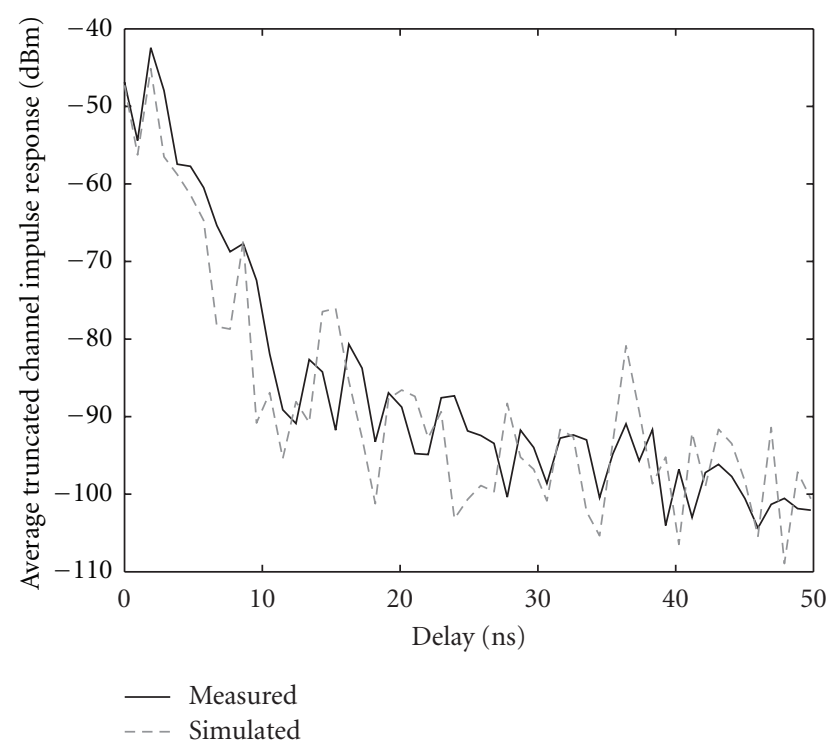

Figure 16: Average measured and simulated channel impulse responses for Rx17 (LOS) in the ISM band.

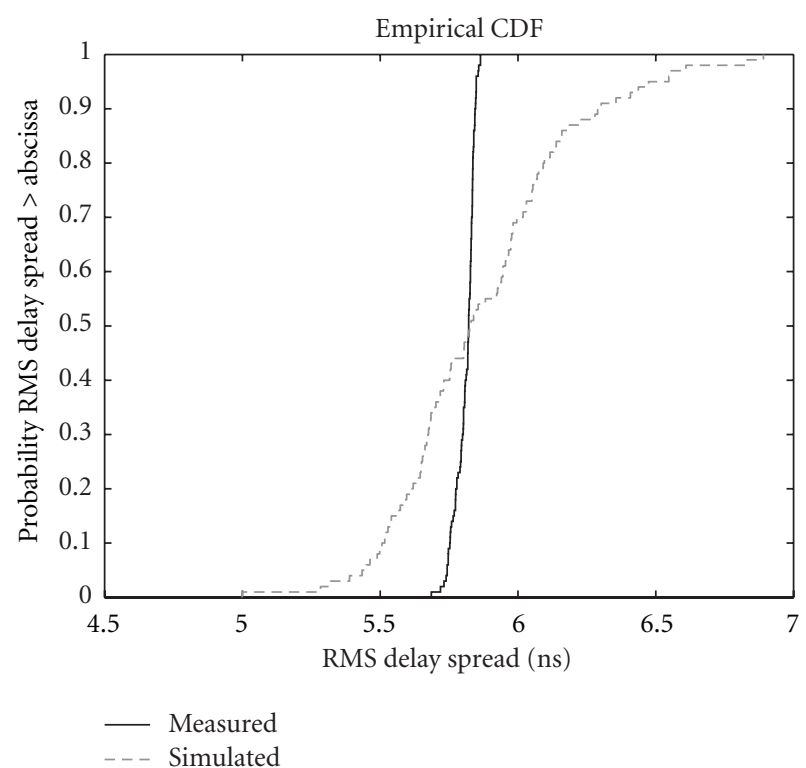

FIGURE 17: CDF of the RMS delay spreads of the measured and simulated channels for Rx17 (LOS) in the UWB band.

the engine is on and off, only the number of the paths are different, especially, in the case of OLOS; that is, the vibration (of the engine) has only the impact on the number of paths, whereas the impact on other channel properties is trivial.

In both frequency ranges, the exponential decay profile was modeled for the path with the corresponding parameters. For the amplitude distribution, many MPCs arrive in the corresponding delay bin in the ISM band, the central limit theorem is therefore valid, and the amplitudes of the delay bins exhibit a Rayleigh (in non-LOS or OLOS case) or Ricean (in LOS case) distributions. On the contrary, only few MPCs fall into each resolvable delay in the UWB band. Therefore, the central limit theorem is not applicable and the amplitudes of the delay bins consequently do not exhibit a Rayleigh or Ricean distribution, but rather log-normal distribution. For the delay dispersion, the RMS delay spread was independent of the LOS/OLOS situations of the channel. Neither of the distance, this is because the cabin space is very small. The MPCs contributing significant energy play a major role in such a small environment if compared to the direct path. This is very important on the selection of the antenna position and the receiver design. For the path loss, the frequency dependency of the path loss in the UWB band was firstly observed. Secondly, the mean path loss increases in the OLOS case and with the presence of the driver. Moreover, it was noted that the mean path loss in the case, when the engine is on, is not very different than in the case, when the engine is off. The measurements obtained were used to estimate the corresponding channel parameters in order to build statistical simulated channel models.

The developed radio channel models give a great additional information to wireless communications designs in a working machine cabin environment.

\section{Acknowledgment}

The authors would like to thank Mr. Ari Isola for his contribution during the measurements. This work was funded by the Finnish Funding Agency for Technology and Innovation through the European Regional Development Fund.

\section{References}

[1] K. Römer and F. Mattern, "The design space of wireless sensor networks," IEEE Wireless Communications, vol. 11, no. 6, pp. 54-61, 2004.

[2] T. Elbatt, C. Saraydar, M. Ames, and T. Talty, "Potential for intra-vehicle wireless automotive sensor networks," in 2006 IEEE Sarnoff Symposium, usa, March 2006.

[3] G. Leen and D. Heffernan, "Vehicles without wires," Computing and Control Engineering Journal, vol. 12, no. 5, pp. 205211, 2001.

[4] AN21E Application Note Human Vibration Measurement EC Directive 2002/44/EC.

[5] D. Cassioli, M. Z. Win, and A. F. Molisch, "The ultra-wide bandwidth indoor channel: from statistical model to simulations," IEEE Journal on Selected Areas in Communications, vol. 20, no. 6, pp. 1247-1257, 2002.

[6] S. S. Ghassemzadeh, R. Jana, C. W. Rice, W. Turin, and V. Tarokh, "Measurement and modeling of an ultra-wide bandwidth indoor channel," IEEE Transactions on Communications, vol. 52, no. 10, pp. 1786-1796, 2004.

[7] W. Niu, J. Li, and T. Talty, "Intra-Vehicle UWB channel measurements and statistical analysis," in 2008 IEEE Global Telecommunications Conference, GLOBECOM 2008, pp. 34023406, usa, December 2008.

[8] W. Xiang, "A vehicular ultra-wideband channel model for future wireless intra-vehicle communications (IVC) Systems," in 2007 IEEE 66th Vehicular Technology Conference, VTC 2007Fall, pp. 2159-2163, usa, October 2007.

[9] http://www.alliancetesteq.com/.

[10] http://tinyurl.com/cyszob/.

[11] http://skycross.com/Products/PDFs/SMT-3TO10M-A.pdf. 
[12] I. Oppermann, M. Hämäläinen, and J. Iinatti, UWB Theory and Applications, John Wiley \& Sons, New York, NY, USA, 2004.

[13] A. Taparugssanagorn, M. Hämäläinen, and J. Iinatti, "UWB and wideband channel models for working machine environment," in Proceedings of the 73rd IEEE Vehicular Technology Conference Spring (VTC'12-Spring), Budapest, Hungary, 2011.

[14] A. Richter, Estimation of radio channel parameters: models and algorithms [Ph.D. thesis], Technische Universitt Ilmenau, Ilmenau, Germany, 2005.

[15] A. Muqaibel, A. Safaai-Jazi, A. Attiya, B. Woerner, and S. Riad, "Path-loss and time dispersion parameters for indoor UWB propagation," IEEE Transactions on Wireless Communications, vol. 5, no. 2, pp. 550-558, 2006.

[16] M. Abramowitz and I. A. Stegun, Handbook of Mathematical Functions, National Bureau of Standards, 1964, Dover Publications, New York, NY, USA, 1965.

[17] C. Tepedelenlioǧlu, A. Abdi, and G. B. Giannakis, "The Ricean K factor: estimation and performance analysis," IEEE Transactions on Wireless Communications, vol. 2, no. 4, pp. 799-810, 2003.

[18] E. Limpert, W. A. Stahel, and M. Abbt, "Log-normal distributions across the sciences: keys and clues," BioScience, vol. 51, no. 5, pp. 341-352, 2001. 

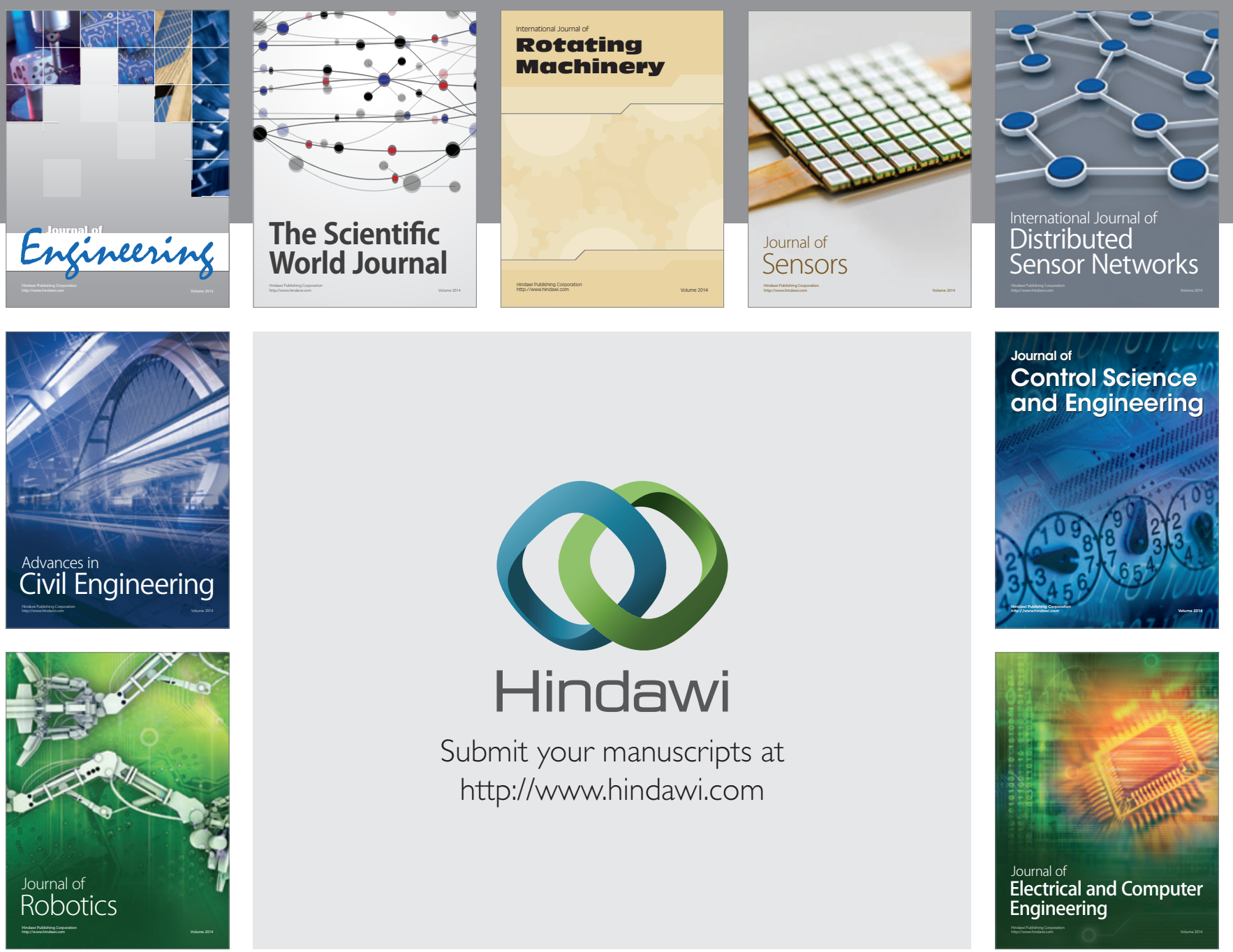

Submit your manuscripts at

http://www.hindawi.com
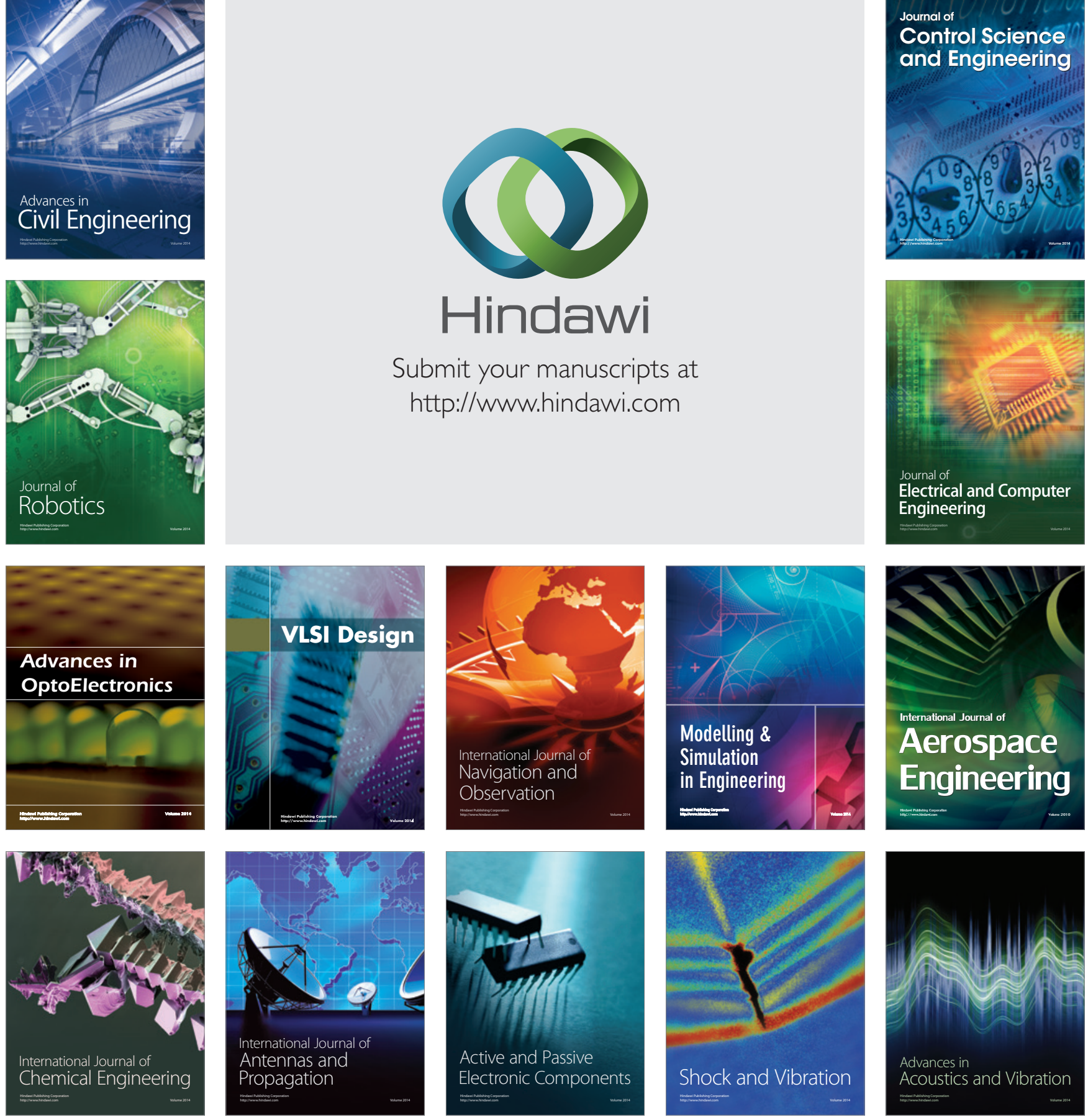